\title{
Метод построения программных средств моделирующих комплексов военного назначения
}

\author{
A.C. Костров 1, адъюнкт, godfatherm69@yandex.ru \\ ${ }^{1}$ Военная академия воздушно-космической обороны им. Г.К. Жукова, \\ 2. Тверь, 170100, Россия
}

Для повышения оперативности и обоснованности принимаемых органами военного управления решений применяются моделирующие комплексы военного назначения, представляющие собой совокупность технических и программных средств, обеспечивающих производство расчетов и имитационного моделирования военных действий. Анализ структуры и функциональных возможностей программных средств современных моделирующих комплексов показал, что на оперативность их применения при использовании по назначению в значительной степени влияет продолжительность ввода исходных данных о действиях потенциального противника. Однако сегодня в общей программной архитектуре моделирующих комплексов, принятых на снабжение в подразделения органов управления, не реализован конкретный технологический инструментарий, позволяющий минимизировать временные затраты на ввод информации в условиях ограниченных сроков применения программных средств.

В статье рассмотрены вопросы построения программных средств в моделирующих комплексах военного назначения, позволяющих сократить временные затраты на ввод исходных данных в процессе моделирования боевых действий в воздушно-космической сфере.

Целью данной статьи является оценка влияния замены действительных значений входных параметров об ударе вероятного воздушно-космического противника значениями по умолчанию на показатели результатов имитационного моделирования боевых действий. Научная новизна заключается в разработке нового метода построения программных средств моделирующих комплексов военного назначения. Приведены числовые примеры расчета значений по умолчанию. Предложен предпочтительный вариант построения программных средств на примере внедрения технологических разработок в общую программную архитектуру моделирующего комплекса.

Ключевые слова: моделирующий комплекс, программные средства, входные параметры, выходные параметры, временные затраты, значения по умолчанию.

Анализ особенностей современных войн и вооруженных конфликтов показывает, что наряду с другими факторами успех боевых действий на стороне тех, кто имеет более эффективную систему управления группировками войск (сил) за счет качества принимаемых решений органами военного управления (ОВУ) на их боевое применение. В настоящее время в целях повышения оперативности и обоснованности принимаемых ОВУ решений применяются моделирующие комплексы военного назначения (MК BН), представляющие собой совокупность технических и программных средств, обеспечивающих расчеты и имитационное моделирование военных (боевых) действий [1].

Результаты анализа применения МК ВН на мероприятиях оперативной подготовки показали, что наряду с достоинствами комплексы имеют и ряд существенных недостатков. Основным из них является значительное превышение нормативных сроков представления результатов имитационного моделирования (выходных параметров) в условиях ограничений применения программных средств МК ВН в соответствии с их назначением выполняемым алгоритмам работы ОВУ [2]. Следствием недостатка является колоссальный объем исходных данных (входных параметров) об обстановке, своих войсках и противнике, который требуется ввести в МК ВН в условиях временных ограничений, отведенных на применение их программных средств.

Анализ структуры и функциональных возможностей программных средств МК ВН показал, что на оперативность их применения при использовании по назначению существенно влияет продолжительность ввода входной информации о действиях вероятного противника [3]. Повысить оперативность применения МК ВН на этапе ввода входной информации можно путем модернизации существующих программных средств: предлагается выбор предпочтительного варианта входных параметров, определяющего состав вводимых параметров и автоматически заменяемых значениями по умолчанию, при ограничениях на 
допустимую ошибку расчета выходных параметров.

Целью данной работы является оценка влияния замены действительных значений входных параметров об ударе вероятного противника значениями по умолчанию на показатели результатов имитационного моделирования боевых действий, направленных на сокращение временных затрат вводимых параметров.

Структурная схема предлагаемого метода построения программных средств МК ВН представлена на рисунке 1. В рамках создания нового научного метода были разработаны представленные далее новые научные методики.

\section{Методика сбора и обработки статистических данных результатов моделирования}

На предварительном этапе для текущего $s$-го варианта моделирования, выбранного для сбора статистики, формируется перечень задействованных входных параметров $X_{i}^{s}-i$-х классов средств воздушно-космического нападения (СВКН) противника. Для данного перечня из БД МК ВН считываются значения по умолчанию $f$-х атрибутов $X_{i}^{s}$, полученные заблаговременно по статистическим данным результатов совокупности реализаций модели $X_{i f}^{\text {умл.ст }}$. Каждый $i$-й входной параметр содержит следующий набор атрибутов, которые могут быть заменены значениями по умолчанию: тип СВКН, номер варианта подвески, режим работы радиоэлектронной станции (РЭС).

В случае отсутствия статистических данных считываются значения, полученные по данным классификаторов моделирующего комплекса $X_{i f}^{\text {Умл.кл }}$, и проводится первый имитационный эксперимент для $s$-го варианта моделирования при значении всех $X_{i}^{s}$, соответствующих значениям по умолчанию [4]. После проведения моделирования формируются и сохраняются значения выходных параметров $Y_{j s}^{\text {умл }}$. В качестве примеров выходных параметров могут рассматриваться количество уничтоженных СВКН противника и объектов прикрытия, расход зенитных управляемых ракет и т.д.

Далее для каждого $X_{i}^{s}$ формируются варианты всех возможных $m$-х сочетаний значений атрибутов var $_{\text {im }}$ с последующим проведением экспериментов по каждому варианту, при этом

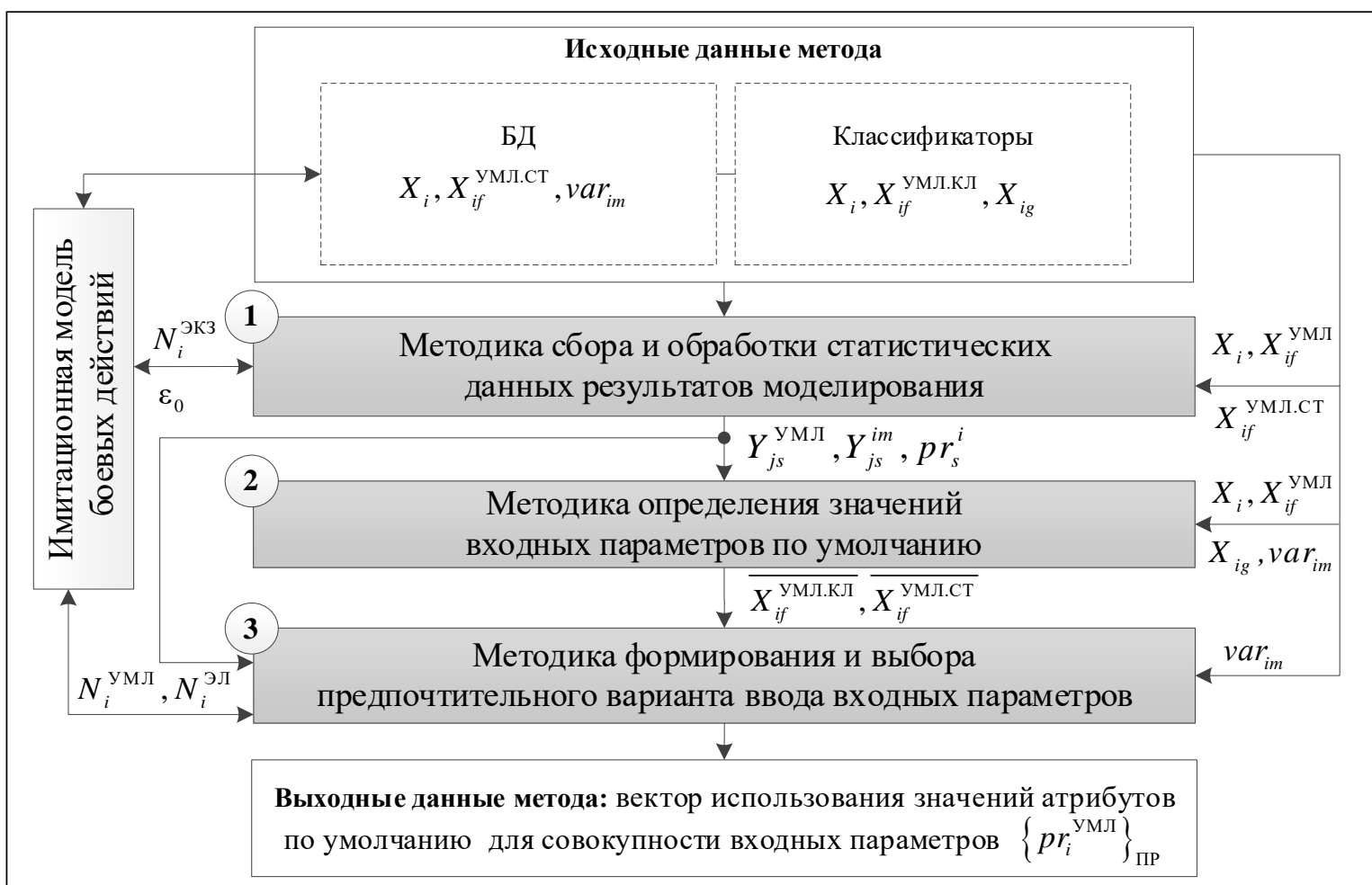

Рис. 1. Структурная схема метода построения программных средств МК ВН

Fig. 1. A block diagram of the method for constructing software military simulation complexes 
значения атрибутов прочих $X_{i}^{s}$ заменяются значениями по умолчанию. По результатам проведенных экспериментов сохраняются новые значения выходных параметров $Y_{j s}^{i m}$.

После экспериментов определяется признак возможности использования статистических данных $p r_{s}^{i}$ каждого варианта моделирования по каждому $X_{i}^{s}: p r_{s}^{i}=0$, если $Y_{j s}^{i 1}=Y_{j s}^{i 2}=\ldots=Y_{j s}^{i N_{m}^{i}}$, $\left(m=\overline{1, N_{m}^{i}}\right)$, где $N_{m}^{i}$ - число вариантов моделирования; $p r_{s}^{i}=1$ - иначе.

Приведенное выражение означает, что $p r_{s}^{i}=0$, если для всех вариантов $v a r_{i m}$ значения хотя бы одного выходного параметра равны между собой. В результате в БД МК ВН остаются только статистические данные, для которых значения всех выходных параметров не равны между собой либо во всех вариантах моделирования значения одного или нескольких выходных параметров будут равны между собой.

\section{Методика определения значений входных параметров по умолчанию}

На предварительном этапе по $i$-му входному параметру для каждого $g$-го атрибута $X_{i g}$, используемого для определения значений по умолчанию, из классификаторов считываются значения атрибутов $\left(X_{i g}\right)_{k g}$, а также перечень вариантов varim, содержащий набор сочетаний значений атрибутов $X_{i f}^{\text {умл.кл }}$. Пусть при определении значений по умолчанию используются атрибуты $X_{i g}: X_{i 1}-$ крейсерская скорость полета СВКН; $X_{i 2}$ - дальность полета; $X_{i 3}$ - высота боевого применения. Пример вариантов значений дальности полета СВКН $X_{i 2}$ приведен в таблице 1.

Далее определяется наличие достоверных статистических данных результатов моделирования для определения значения по умолчанию. При их наличии значение по умолчанию определяется результатами моделирования, в противном случае - данными из классификаторов. Тогда значения по умолчанию определяются исходя из минимизации суммарного отклонения значений атрибутов $X_{i g}$ от медиан этих атрибутов, в том числе $X_{i g}: M e\left(X_{i g}\right)$. Для каждого варианта $v a r_{i m}$ определяются значения атрибутов $X_{i g}$ с учетом вложенной структуры входного параметра.

Пусть в классификаторах имеются данные, приведенные в таблице 2.

По представленным структурам $X_{i g}$ формируются варианты сочетания значений атрибутов. Для каждого варианта рассчитывается суммарное относительное отклонение значений $\left(X_{i g}\right)_{m}$ атрибутов $X_{i g}$ от медиан $M e\left(X_{i g}\right)$ :

$$
\Delta_{i m}=\sum_{m}\left|\frac{\left(X_{i g}\right)_{m}-M e\left(X_{i g}\right)}{M e\left(X_{i g}\right)}\right| .
$$

Для рассмотренного выше примера каждое суммарное относительное отклонение представлено в таблице 3 .

\section{Пример вариантов числовых значений дальности полета СВКН}

Таблица 1

An example of options for the numerical values of the aircraft flight range

\begin{tabular}{|c|c|c|}
\hline Номер варианта & Атрибут входного параметра & Значение атрибута \\
\hline 1 & Дальность полета F-15 с вариантом подвески № 1 & $\left(X_{i 2}\right)_{1}=650$ км \\
\hline 2 & Дальность полета F-15 с вариантом подвески № 2 & $\left(X_{i 2}\right)_{2}=1000$ км \\
\hline 3 & Дальность полета F-16 с вариантом подвески № 1 & $\left(X_{i 2}\right)_{3}=950$ км \\
\hline 4 & Дальность полета F-16 с вариантом подвески № 2 & $\left(X_{i 2}\right)_{4}=900$ км \\
\hline
\end{tabular}

Таблица 2

Пример вложенной структуры входного параметра

Table2

An example of a nested input parameter structure

\begin{tabular}{|c|c|c|c|c|}
\hline Тип СВКН & \multicolumn{2}{|c|}{ F-15 } & \multicolumn{2}{|c|}{ F-16 } \\
\hline Крейсерская скорость полета $X_{i 1}$, км/ч & \multicolumn{2}{|c|}{1200} & \multicolumn{2}{|c|}{1300} \\
\hline Вариант подвески & \multicolumn{2}{|c|}{ № 1} & \multicolumn{2}{|c|}{ № 2} \\
\hline Дальность полета $X_{i 2}$, км & 650 & 950 & 1000 & 900 \\
\hline Высота боевого применения $X_{i 3}$, км & 350 & 450 & 550 & 250 \\
\hline
\end{tabular}


Пример вариантов сочетаний значений атрибутов по умолчанию

An example of default attribute value combinations

An example of default attribute value combinations

\begin{tabular}{|c|c|c|c|c|}
\hline Вариант & $\left(\boldsymbol{X}_{\boldsymbol{i 1}}\right)_{\boldsymbol{i}}, \mathbf{K M} / \mathbf{Y}$ & $\left(\boldsymbol{X}_{\boldsymbol{i 2}}\right)_{\boldsymbol{i}}, \mathbf{K M}$ & $\left(\boldsymbol{X}_{\boldsymbol{i 3}}\right)_{\boldsymbol{i}}, \mathbf{K M}$ & $\boldsymbol{\Delta}_{\boldsymbol{i \mathbf { m }}}$ \\
\hline var $_{i 1}$ & 1200 & 650 & 350 & 0,462 \\
\hline var $_{i 2}$ & 1200 & 1000 & 550 & 0,496 \\
\hline var $_{\mathbf{3}}$ & 1300 & 950 & 450 & 0,192 \\
\hline $\operatorname{var}_{i 4}$ & 1300 & 900 & 250 & 0,442 \\
\hline $\operatorname{Me}\left(X_{i 1}\right)$ & 1250 & 925 & 400 & \\
\hline
\end{tabular}

Далее определяется вариант с минимальным значением отклонения: $\operatorname{var}_{i}^{\text {умл }}=\arg \min _{m} \Delta_{i m}$. Избранному варианту соответствует определенное сочетание значений атрибутов $\left(X_{i f}^{\text {умЛ }}\right)_{k f}$, которые фиксируются как новые

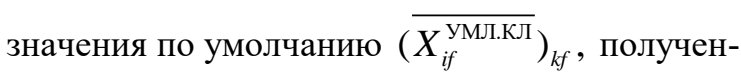
ные по данным классификаторов, и заносятся в БД моделирующего комплекса.

Для числового примера, рассмотренного в таблице 2 , сочетания значений по умолчанию соответственно равны: $\left(X_{i 1}^{\mathrm{yM}}\right)_{2}+\left(X_{i 2}^{\text {УМЛ })_{3}-}\right.$ F-16 с вариантом подвески № $1 \mathrm{~F}-16 ; X_{i 1}^{\text {умл }}$

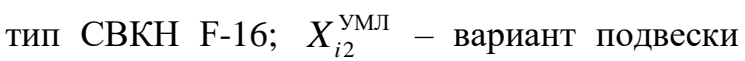
№ 1 .

Следующий этап данной методики - установление значения по умолчанию с использованием статистических данных $X_{i f}^{\text {умл.ст }}$, определяемых исходя из минимизации суммарного отклонения значений $j$-х выходных параметров $Y_{j}^{\text {im }}$ варианта var $_{\text {im }}$ сочетаний значений атрибутов $X_{i f}^{\text {умл }}$ от значений выходных параметров $Y_{j}^{i k}$ всех других вариантов $v a r_{i k}$.

Пусть имеются следующие выходные данные: $Y_{1}^{i 1}$ - число уничтоженных СВКН; $Y_{2}^{i 1}-$ число уничтоженных объектов прикрытия; $Y_{3}^{i 1}$ - расход зенитных управляемых ракет. Для входного параметра имеются четыре варианта $\operatorname{var}_{\text {im }}$ сочетания значений атрибутов $X_{i f}^{\text {умл }}$ (табл. 3), также по результатам моделирования получены значения выходных параметров, приведенные в таблице 4.

Так, для каждого $m$-го варианта рассчитывается суммарное отклонение значений $j$-го выходного параметра конкретного варианта от значений конкретного выходного параметра всех других вариантов: $\Delta_{j}^{i m}=\sum_{k}\left|\frac{Y_{j}^{i m}-Y_{j}^{i k}}{Y_{j}^{i \max }}\right|, \quad$ где
$Y_{j}^{i \max }=\max _{m} Y_{j}^{i \mathrm{~m}}-$ максимальное значение выходного параметра по всем вариантам varim. Отклонения по каждому варианту сведены в таблицу 5.

\section{Пример числовых значений выходных параметров}

Таблица 4

Table 4

An example of numeric output parameters

\begin{tabular}{|c|c|c|c|}
\hline Вариант & $\boldsymbol{Y}_{\mathbf{1}}^{\boldsymbol{i 1}}$ & $\boldsymbol{Y}_{\mathbf{2}}^{\boldsymbol{i 1}}$ & $\boldsymbol{Y}_{\mathbf{3}}^{\boldsymbol{i 1}}$ \\
\hline $\operatorname{var}_{i 1}$ & 24 & 3 & 35 \\
\hline $\operatorname{var}_{i 2}$ & 21 & 4 & 39 \\
\hline $\operatorname{var}_{i 3}$ & 15 & 7 & 26 \\
\hline $\operatorname{var}_{i 4}$ & 19 & 6 & 31 \\
\hline
\end{tabular}

Промежуточный этап методики состоит в следующем. Для каждого m-го варианта рассчитывается суммарное отклонение по всем выходным параметрам: $\Delta_{i m}=\sum_{j} w_{j} \Delta_{j}^{i m}$, где $w_{j}$ - весовой коэффициент $j$-го выходного параметра, рассчитанный с помощью элементов метода анализа иерархии [5].

Таблииа 5

Пример суммарных отклонений значений выходного параметра

Table 5

An example of the total deviations of the output parameter values

\begin{tabular}{|c|c|c|c|c|}
\hline Вариант & $\Delta_{\mathbf{1}}^{i m}$ & $\mathbf{\Delta}_{\mathbf{2}}^{\text {im }}$ & $\boldsymbol{\Delta}_{\mathbf{3}}^{\text {im }}$ & $\boldsymbol{\Delta}_{\text {im }}$ \\
\hline $\operatorname{var}_{i 1}$ & 0,708 & 1,143 & 0,436 & 0,844 \\
\hline $\operatorname{var}_{i 2}$ & 0,458 & 0,857 & 0,641 & 0,712 \\
\hline $\operatorname{var}_{i 3}$ & 0,792 & 1,143 & 0,692 & $\mathbf{0 , 9 3 8}$ \\
\hline var $_{i 4}$ & 0,458 & 0,857 & 0,436 & 0,651 \\
\hline
\end{tabular}

В качестве примера для расчета суммарного отклонения для каждого т-го варианта взяты следующие значения весовых коэффициентов: $w_{1}=0,2, w_{2}=0,5, w_{3}=0,3$. Численные значения суммарного отклонения $\Delta_{i m}$ по каждому выходному параметру представлены в таблице 5.

Заключительный этап методики состоит в том, что для выбора варианта var $_{\text {im }}$ с минималь- 
ным отклонением рассчитывается верхняя граница доверительного интервала: $\Delta_{i m}^{S_{X}}=\overline{\Delta_{i m}}+$ $+t_{\beta} \sigma_{i m}$, где $\overline{\Delta_{i m}}$ и $\sigma_{i m}-$ оценка математического ожидания и среднеквадратического суммарного взвешенного отклонения для каждого варианта соответственно; $t_{\beta}-$ коэффициент Стьюдента для заданной доверительной веро-

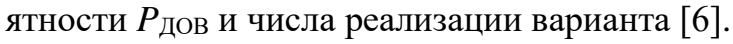

В итоге определяется вариант, имеющий минимальное значение отклонения: $\operatorname{var}_{i}^{\text {умл }}=$ $=\arg \min _{m} \Delta_{i m}^{S_{X}}$. Избранному варианту соответствует определенное сочетание значений атри-

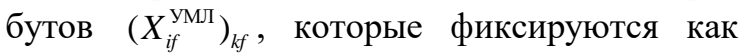
новые значения по умолчанию $\left(\overline{X_{i f}^{\text {умл.ст }}}\right)_{k f}$, полученные по статистическим данным, и заносятся в БД.

\section{Методика формирования и выбора предпочтительного варианта ввода входных параметров}

На предварительном этапе вычисляется среднее значение ошибки определения $Y_{j s}^{i m}$ для совокупности вариантов в $s$-х реализациях модели [7]: $\varepsilon_{j s}^{i}=\frac{1}{N_{m}^{i}} \sum_{m}\left|\frac{Y_{j s}^{i m}-Y_{j s}^{\text {УМЛ }}}{Y_{j s}^{i \max }}\right|$.

Затем рассчитывается взвешенная суммарная ошибка по совокупности выходных параметров $Y_{j s}^{i m}$ в $s$-х реализациях: $\varepsilon_{s}^{i}=\sum_{j} w_{j} \varepsilon_{j s}^{i}$. Далее по совокупности $s$-х реализаций модели определяются математическое ожидание $\bar{\varepsilon}_{i}$ и дисперсия $\sigma_{\varepsilon_{i}}^{2}$ ошибки расчета выходных параметров, а также верхняя граница значения ошибки: $\varepsilon_{i}^{\text {sup }}=\bar{\varepsilon}_{i}+t_{\beta} \sigma_{\varepsilon_{i}}$.

Для соблюдения условия ввода данных в условиях временных ограничений определяются параметры закона распределения времени ввода. К ним относятся математическое ожидание $\bar{t}_{\text {эл }}$ и дисперсия $\sigma_{\text {эл }}^{2}$ времени выполнения одной элементарной операции [8]. Кроме того, каждый вариант характеризуется своим значением вектора признаков использования значений атрибутов по умолчанию $\left\{p r_{i}^{\text {умл }}\right\}$.

Рассчитываются математическое ожидание и дисперсия времени ввода входных параметров каждого варианта соответственно:
$\overline{T^{\Sigma}}=\overline{t_{\text {Эл }}}\left[N_{\text {ПР }}+\sum_{i}\left(1-p r_{i}^{\text {уМЛ }}\right) N_{i}^{\text {ЭКЗ }} N_{i}^{\text {ВО }}+\right.$

$\left.+\sum_{i} p r_{i}^{\text {уМЛ }} N_{i}^{\text {ЭК } 3} N_{i}^{\text {УМЛ }}\right]$,

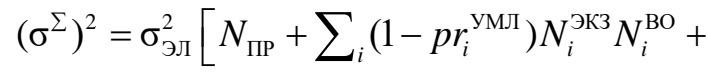

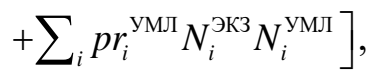

где $N_{П р}$ - число предварительных элементар-

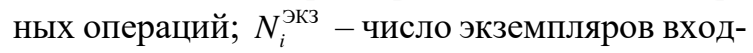
ного параметра; $N_{i}^{\text {умл }}$ - число элементарных операций для ввода входного параметра при задании значений по умолчанию.

Далее для каждого варианта вычисляются суммарная ошибка расчета выходных параметров и верхняя граница времени ввода:

$$
\begin{gathered}
\varepsilon=\sum_{i=1}^{N_{x}} p r_{i}^{\text {умл }} \varepsilon_{i}^{\text {sup }}, \\
T^{\text {sup }}=\overline{T^{\Sigma}}+t_{\beta} \sigma^{\Sigma} .
\end{gathered}
$$

Поиск предпочтительного варианта ввода данных определяется исходя из выполнения условий $\varepsilon \leq \varepsilon_{0}, T^{\text {sup }} \rightarrow \min$. Результат поиска предпочтительный вектор признаков использования значений атрибутов по умолчанию для совокупности входных параметров $\left\{p r_{i}^{\text {умл }}\right\}_{\Pi \text { п }}$.

Разработана программа для формирования входных параметров, реализующая предлагаемый метод [9]. Структура макета программы содержит перечень соответствующих программных модулей, представленных на рисунке 2.

На рисунке 3 приведена структурная схема дополнительной БД, включающей в себя объекты и их атрибуты, необходимые для реализации программы [10].

Представленные таблицы связаны с имеющимися классификаторами моделирующего

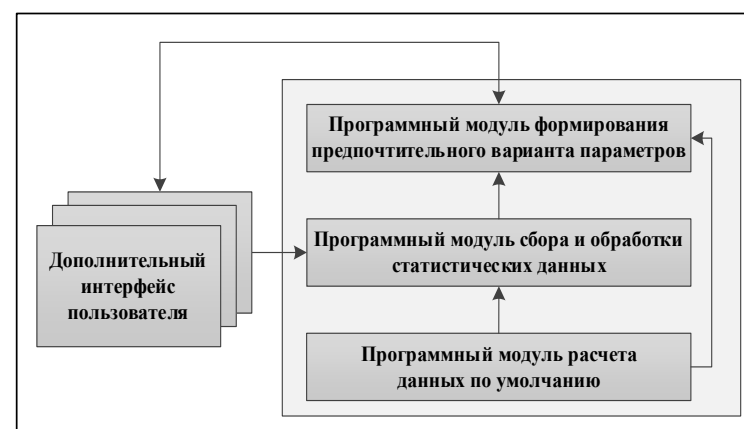

Рис. 2. Макет программы для формирования входных параметров

Fig. 2. The layout of the program for forming input parameters 
комплекса: типы СВКН, варианты подвески типов СВКН, типы РЭС и режимы работы РЭС.

Апробация предлагаемого метода и имитационные эксперименты были проведены на базе Военной академии воздушно-космической обороны (г. Тверь) с использованием многофункционального МК ВН «Небосвод 2.0», позволяющего моделировать боевые действия противоборствующих группировок в воз- душно-космической сфере и оценивать их результаты в едином интерактивном пространственно-временном контуре управления. Программные средства комплекса разработаны в программно-технической среде Microsoft Visual Studio 2015 (язык программирования C++, СУБД PostgreSQL) [11].

На рисунке 4 представлен предпочтительный вариант построения программных средств,

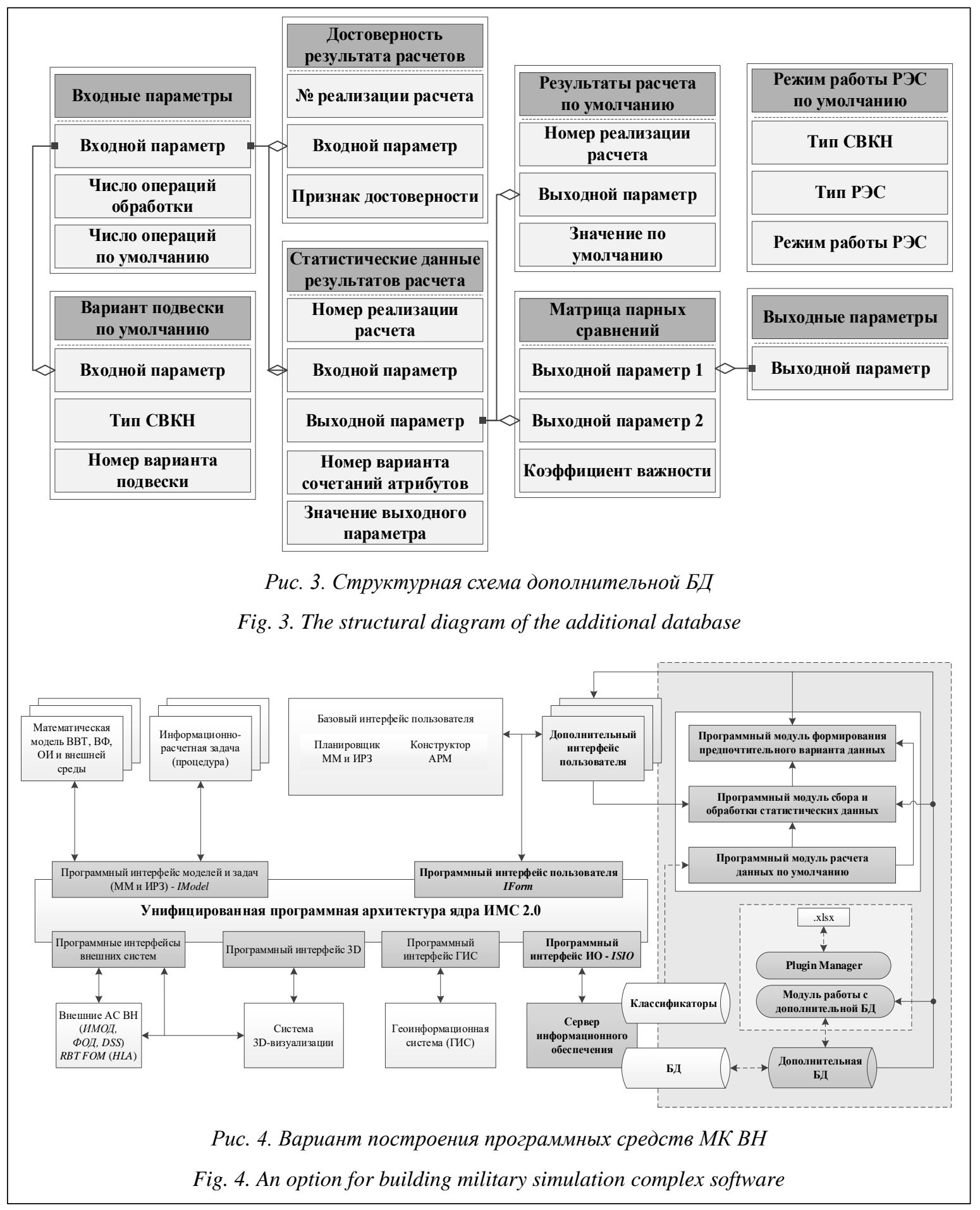


предполагающих внедрение технологических решений в универсальную программную архитектуру МК ВН «Выпускник-ВАГШ» (НПО «РусБИТех», г. Тверь), что позволит организовать дальнейшие разработки (модернизацию) перспективных программных средств на единой методической и технологической основе с учетом их использования по назначению.

\section{Заключение}

Таким образом, установлено непосредственное влияние замены действительных значений входных параметров значениями по умолчанию на показатели результатов имитационного моделирования боевых действий. Следовательно, для сокращения временных затрат на ввод входных параметров необходимо модернизировать программные средства технологическими разработками таким образом, чтобы с их помощью обеспечить соответ- ствие временных сроков, отведенных на ввод исходных данных, регламентированных нормативными документами, фактическим временным затратам, при этом не превысив допустимую ошибку расчета выходных параметров (результатов моделирования).

Научная новизна исследования заключается в разработке метода построения программных средств МК ВН, отличающегося от существующих тем, что за счет нового построения программных средств осуществляется выбор варианта данных, определяющего состав вводимых параметров и автоматически заменяемых значениями по умолчанию.

Предложенный метод может быть использован при моделировании боевых действий в воздушно-космической сфере с использованием программных средств МК ВН по назначению при подготовке и непосредственно в процессе боевых действий.

\title{
Литература
}

1. Тиханычев О.В. Субъективные аспекты применения математического моделирования военных действий в практике работы органов военного управления // Военная мысль. 2011. № 10. С. 49-53.

2. Лясковский В.Л., Ризаев Р.Н., Допира Р.В., Кучеров Ю.С. Методика оценки степени влияния модели системы управления на эффективность применения частей и подразделений радиоэлектронной борьбы в моделирующих комплексах военного назначения // Вопросы радиоэлектроники. 2020. № 10 . C. 46-52. DOI: 10.21778/2218-5453-2020-10-46-52.

3. Голубев Ю.Н., Каргин В.Н. Информационные технологии в управлении войсками // Военная мысль. 2005. № 6. С. 43-45.

4. Созинов П.А., Глушков И.Н. Имитационное моделирование боевых действий: теория и практика. Тверь, 2013. 528 с.

5. Иванов В.К., Думина Д.С., Семенов Н.А. Определение весовых коэффициентов для аддитивной фитнес-функции генетического алгоритма // Программные продукты и системы. 2020. Т. 33 . № 1. C. 47-53. DOI: 10.15827/0236-235X.129.047-053.

6. Бусленко Н.П. Моделирование сложных систем. М.: Наука, 1978. 400 с.

7. Вентцель Е.С. Теория вероятностей; [пер. с англ.]. М: ЮСТИЦИЯ, 2018. 658 с.

8. Шеннон Р. Имитационное моделирование систем - искусство и наука. М.: Мир, 1978. 420 с.

9. Девятков В.В. Методология и технология имитационных исследований сложных систем: современное состояние и перспективы развития. М.: ИНФРА-М, 2013. 448 с.

10. Шлее M. Qt 5.10. Профессиональное программирование на С++. СПб: БХВ-Петербург, 2018. $1072 \mathrm{c}$.

11. Костров А.С. К вопросу совершенствования программных средств моделирования автоматизированных систем военного назначения // Состояние и перспективы развития математического моделирования сложных процессов в системах военного и двойного назначения: сб. матер. конф. Анапа, 2020. С. $247-255$.

\section{The method of constructing software for military simulation complexes}

\author{
A.S. Kostrov ${ }^{1}$, Adjunct, godfatherm69@yandex.ru \\ ${ }^{1}$ Military Academy of the Aerospace Defence, Tver, 170022, Russian Federation
}

Abstract. Nowadays, in order to increase the efficiency and validity of decisions taken by military command and control bodies, there are military simulation complexes. They are a combination of hardware and 
software that ensure calculations and simulation of military operations. The analysis of the structure and functionality of the software of modern modeling complexes has shown that their efficiency when used for their intended purpose is largely affected by the duration of input of initial data on potential adversary's actions. However, in the general software architecture accepted for supply to the subdivisions of the control bodies, modeling complexes, a specific technological toolkit that allows minimizing the time spent on information input under the conditions of limited periods of software application has not been implemented.

The paper discusses the issues of building software in military simulation complexes, which make it possible to reduce the time spent on entering initial data in the process of simulating combat operations in the aerospace sphere.

The purpose of this paper is to assess the effect of replacing the actual values of the input parameters about the strike of a probable aerospace enemy with default values on the indicators of the combat operation simulation results. Scientific novelty is the development of a new method for constructing software for military simulation complexes. There are numerical examples of calculating default values provided. The preferred option for building software is proposed on the example of introducing technological developments into the general software architecture of the modeling complex.

Keywords: modeling complex, software, input parameters, output parameters, time costs, default values.

\section{References}

1. Tikhanychev O.V. Subjective aspects of using mathematical modeling of military operations in the military command and control practice. Military Thought, 2011, no. 10, pp. 49-53 (in Russ.).

2. Lyaskovskiy V.L., Rizaev R.N., Dopira R.V., Kucherov Yu.S. Methodology for assessing the influence of control system on efficiency of application of electronic warfare units in military modeling complexes. Issues of Radio Electronics, 2020, no. 10, pp. 46-52. DOI: 10.21778/2218-5453-2020-10-46-52 (in Russ.).

3. Golubev Yu.N., Kargin V.N. Information technology in the management of troops. Military Article, 2005, no. 6, pp. 43-45 (in Russ.).

4. Sozinov P.A., Glushkov I.N. Simulation of Combat Operations: Theory and Practice. Tver, 2013, 528 p. (in Russ.).

5. Ivanov V.K., Dumina D.S., Semenov N.A. Determination of weight coefficients for additive fitness function of genetic algorithm. Software and Systems, 2020, vol. 33, no. 1, pp. 47-53. DOI: 10.15827/0236235X.129.047-053 (in Russ.).

6. Buslenko N.P. Modeling Complex Systems. Moscow, 1978, 400 p. (in Russ.).

7. Wentzel E.S. Probability Theory. Moscow, 2018, 658 p. (in Russ.).

8. Shannon R.E. Systems Simulation: The Art and Science. Prentice Hall, 1975, 387 p. (Russ. ed.: Moscow, 1978,420 p.).

9. Devyatkov V.V. Methodology and Technology of Simulation Studies of Complex Systems: Current State and Prospects of Development. Moscow, 2013, 448 p. (in Russ.).

10. Shlee M. Qt 5.10 Professional Programming on C++. St. Petersburg, 2018, 1072 p. (in Russ.).

11. Kostrov A.S. On the issue of improving software tools for modeling automated systems for military use. Conf. Proc. State and Development Prospects of Mathematical Modeling of Complex Processes in Military and Dual-Use Systems. Anapa, 2020, pp. 247-255 (in Russ.).

\section{Для цитирования}

Костров А.С. Метод построения программных средств моделирующих комплексов военного назначения // Программные продукты и системы. 2021. Т. 34. № 3. С. 440-447. DOI: $10.15827 / 0236-235 X .135 .440-447$.

\section{For citation}

Kostrov A.S. The method of constructing software for military simulation complexes. Software \& Systems, 2021, vol. 34, no. 3, pp. 440-447 (in Russ.). DOI: 10.15827/0236-235X.135.440-447. 